Instituto Internacional de Investigación y Desarrollo Tecnológico Educativo INDTEC, C.A.

DOI: https://doi.org/10.29394/Scientific.issn.2542-2987.2020.5.15.8.168-190

OAI-PMH: http://www.indteca.com/ojs/index.php/Revista Scientific/oai

Artículo Original / Original Article

\title{
Los estudios de pertinencia desde referentes contextuales: Experiencia desde una maestría en Educación Inclusiva
}

\author{
Autores: José Ignacio Herrera Rodríguez \\ Universidad Nacional de Educación, UNAE \\ joseighr2015@gmail.com \\ Azogues, Ecuador \\ https://orcid.org/0000-0002-4750-3655 \\ Geycell Emma Guevara Fernández \\ Universidad Nacional de Educación, UNAE \\ geycellgf@gmail.com \\ Azogues, Ecuador \\ https://orcid.org/0000-0003-4872-8431 \\ Graciela de la Caridad Urías Arbolaez \\ Universidad Nacional de Educación, UNAE \\ gracielauriasarbolaez@yahoo.es \\ Azogues, Ecuador \\ https://orcid.org/0000-0001-5927-3351
}

\section{Resumen}

Los estudios de pertinencia y prospectiva se consideran totalmente necesarios en los procesos de diseño de carreras universitarias y programas de postgrados, los que deben tomar en cuenta referentes teóricos y contextuales sobre el área del saber para el que se haga la propuesta. El objetivo de este trabajo es presentar la evaluación de pertinencia realizada para la maestría en Educación Inclusiva de la Universidad Nacional de Educación de Ecuador, en un primer momento el análisis de las normativas que respaldaban la propuesta y en un segundo momento un análisis contextual, para el que se seleccionó una muestra que respondía al criterio de identificar el conocimiento que tenían los profesionales acerca de la educación inclusiva, la información sobre la necesidad de superación profesional en este campo del saber, las expectativas de mayores oportunidades de empleo y desarrollo profesional a partir de la maestría, esta información se recopiló a través de encuestas y grupos de discusión, lo que permitió identificar las demandas del profesorado, el diseño curricular deseado, las competencias que se debían formar con la instrumentación del programa de maestría y el perfil de los egresados de dicha programa.

Palabras clave: formación de docentes; profesor especializado; pertinencia de la educación.

Cómo citar este artículo:

Herrera, J., Guevara, G., \& Urías, G. (2020). Los estudios de pertinencia desde referentes contextuales: Experiencia desde una maestría en Educación Inclusiva. Revista Scientific, 5(15), 168-190, e-ISSN: 2542-2987. Recuperado de: https://doi.org/10.29394/Scientific.issn.25422987.2020.5.15.8.168-190

Fecha de Recepción: 08-10-2019
Fecha de Aceptación: 12-12-2019
Fecha de Publicación: 05-02-2020 


\title{
Relevance studies from contextual references: Experience from a master's degree in Inclusive Education
}

\begin{abstract}
Relevance and prospective studies are considered totally necessary in the design processes of university careers and postgraduate programs, which must take into account theoretical and contextual references on the area of knowledge for which the proposal is made. The objective of this work is to present the relevance assessment carried out for the Master's degree in Inclusive Education of the National University of Education of Ecuador, at first the analysis of the regulations that supported the proposal and in a second moment a contextual analysis, to the one that selected a sample that responded to the criterion of identifying the knowledge that professionals had about inclusive education, information about the need for professional improvement in this field of knowledge, the expectations of greater employment opportunities and professional development from the master's degree, this information was collected through surveys and discussion groups, which allowed to identify the demands of the teaching staff, the desired curricular design, the competences that should be formed with the instrumentation of the master's program and the profile of the graduates of said program.
\end{abstract}

Keywords: teacher education; special education teachers; educational relevance.

\section{How to cite this article:}

Herrera, J., Guevara, G., \& Urías, G. (2020). Relevance studies from contextual references: Experience from a master's degree in Inclusive Education. Revista Scientific, 5(15), 168-190, eISSN: 2542-2987. Recovered from: https://doi.org/10.29394/Scientific.issn.2542-2987.2020.5.15.8.168190

Date Received: 08-10-2019
Date Acceptance:

12-12-2019
Date Publication: 05-02-2020 


\section{Introducción}

Las demandas que la sociedad realiza a las universidades son cada vez más crecientes, tanto para la incorporación de nuevas carreras de pregrado, como para los programas de postgrados. Ante esta realidad se pueden encontrar varias barreras, una de ellas está relacionada con los estudios de las necesidades y demandas para diseñar las respuestas que respondan a las demandas sociales que le dieron origen.

La Organización de las Naciones Unidas para la Educación, la Ciencia y la Cultura (UNESCO, 1995a): enfatiza en "mejorar la pertinencia de la educación superior respondiendo a los desafíos de un mundo cambiante en los planos internacional, regional, nacional y comunitario" (pág. 48).

La Oficina Regional de Educación para América Latina y el Caribe y la Organización de las Naciones Unidas para la Educación, la Ciencia y la Cultura (OREALC/UNESCO, 2013): destaca la necesidad de "regular la pertinencia de la oferta de postgrados" (pág. 133). Coinciden con esta idea los trabajos de Darling-Hammond (2006); y Crowe (2010): donde se enfatiza en la necesidad de desarrollar criterios e indicadores para la construcción de programas y mallas que respondan a estudios de pertinencia e impacto en el área para la que son diseñados.

Para la Organización de las Naciones Unidas para la Educación, la Ciencia y la Cultura (UNESCO, 1998):

La pertinencia de la educación superior debe evaluarse en función de la adecuación entre lo que la sociedad espera de las instituciones y lo que éstas hacen. Ello requiere normas éticas, imparcialidad política, capacidad crítica y, al mismo tiempo, una mejor articulación con los problemas de la sociedad y del mundo del trabajo, fundando las orientaciones a largo plazo en objetivos y necesidades societales, comprendidos el respeto de las culturas y la protección del medio ambiente (pág. 24).

En tal sentido, en este trabajo se presenta el estudio de pertinencia 
realizado para diseñar la maestría en Educación Inclusiva de la Universidad Nacional de Educación de Ecuador, centrado en el estudio de campo, para conocer la opinión de docentes y directivos, así como el estado de la problemática y llegar a las demandas del profesorado, las competencias curriculares y el perfil de los egresados de dicho programa.

\section{Los estudios de pertinencia de los programas educativos}

\subsection{Consideraciones teóricas}

Según Burgos, Rodríguez y López (2011):

La evaluación de la pertinencia de los programas educativos alude al análisis de la capacidad de la Universidad para responder a las expectativas de la comunidad. En términos más amplios, estudia aspectos referentes a las prioridades establecidas por los planes de desarrollo; estudios de oferta y demanda educativa; y estudios de competencia y de formación integral el estudiante, así como de los mecanismos de producción y distribución del conocimiento (pág. 7).

La pertinencia en los programas de estudio ya sean de pre o postgrado se asocian a la relación y coherencia que debe existir entre la propuesta que se presenta y las necesidades identificadas en el desarrollo social y económico del entorno en el que se insertará el programa, los estudios de pertinencia permiten asignar significación y valor a la propuesta.

La pertinencia, se erigirá como una cualidad que caracterice los vínculos de la universidad con la sociedad, donde la universidad sea reconocida por su rol transformador, por hacer propuestas innovadoras que resuelvan los principales problemas de la sociedad, coherente con esto, considerar la misión y funciones de cada uno de los programas de estudio, los modos de gestionar la docencia y la investigación, el vínculo con la colectividad abogando por ser más equitativa y responsable por el bienestar de todos, así como oportuna al ajustarse a las realidades de cada país. 
En la Conferencia regional, auspiciada por el Centro Regional para la Educación Superior en América Latina y el Caribe Regional y la Organización de las Naciones Unidas para la Educación, la Ciencia y la Cultura (CRESALC/UNESCO), Didriksson, citado por Albornoz (1996), establece que:

Para que las universidades e instituciones de educación superior de América Latina y el Caribe, puedan desempeñar un papel protagónico en el nuevo proceso de desarrollo que están construyendo sus sociedades, se requiere diseñar una estrategia de cambio, que pueda ser correspondida con acciones inmediatas de amplia cobertura y extensión a nivel continental. Esto no podrá realizarse sin una adecuada cooperación Internacional (pág. 7).

La Organización de las Naciones Unidas para la Educación, la Ciencia y la Cultura (UNESCO, 1995b), en una visión de las respuestas de la educación superior plantea:

La pertinencia de la educación superior se considera primordialmente en función de su cometido y su puesto en la sociedad, de sus funciones con respecto a la enseñanza, la investigación y los servicios conexos, y de sus nexos con el mundo del trabajo en sentido amplio, con el Estado y la financiación pública y sus interacciones con otros niveles y formas de educación (pág. 8).

Por su parte, Tünnermann (2006), afirma que:

La pertinencia tiene que ver con el "deber ser" de las Universidades, es decir, con una imagen deseable de las mismas. Un "deber ser", por cierto, ligado a los grandes objetivos, necesidades y carencias de la sociedad en que ellas están insertas y a los retos del nuevo contexto mundial (pág. 1).

Según García (1996), citada por Malagón (2003), la pertinencia es considerada como un aspecto determinante en la calidad: "ese tejido de relaciones y su correspondiente campo de pertinencia garantizan que el criterio de calidad utilizado sea endógeno a esa sociedad, en la medida que 
refleja las prioridades por ella sancionadas, aún de forma difusa e inconsciente" (pág. 130).

En esencia, según Martínez, Rodríguez y García (2018), la pertinencia debe responder a: "...lograr un equilibrio en la relación universidad instituciones escolares, desde valores trascendentales como la igualdad, equidad, solidaridad y el respeto a la diversidad..." (pág. 161).

Como se puede apreciar, existe coincidencia en lo declarado por los organizamos internacionales y por los autores consultados, pues hablar de pertinencia implica hacer referencia al rol transformador que deben jugar las universidades a la hora de ofrecer programas de formación que resuelvan los problemas acuciantes de la sociedad y donde se introduzcan los principales aportes que en el campo de la investigación se generen.

\section{Los estudios de pertinencia para los planes y programas de estudio en Ecuador}

Dentro del marco legal ecuatoriano, los estudios de pertinencia encuentran su base jurídica y política desde la Constitución de la Republica del Ecuador (2008):

El sistema de educación superior estará articulado al sistema nacional de educación y al Plan Nacional de Desarrollo; la ley establecerá los mecanismos de coordinación del sistema de educación superior con la Función Ejecutiva. Este sistema se regirá por los principios de autonomía responsable, cogobierno, igualdad de oportunidades, calidad, pertinencia, integralidad, autodeterminación para la producción del pensamiento y conocimiento (art. 351).

Por su parte, en la Ley Orgánica de Educación Superior (LOES, 2018): se expresa que en "...las instituciones de educación superior articularán su oferta docente, de investigación y actividades de vinculación con la sociedad, a la demanda académica, a las necesidades..." (art. 107). 
En lo que respecta al nivel de formación de los docentes, según los datos obtenidos en agosto del año 2015 desde el Ministerio de Educación, solo el $13 \%$ de los docentes del magisterio fiscal tenían un título de cuarto nivel, lo que implica una cifra sumamente baja en relación a otros países.

El $13 \%$ de docentes que tenían cuarto nivel, equivalente a 20.636 docentes de ellos el $93 \%$ contaban con un título que estaba relacionado a las Ciencias de la Educación, sin embargo, el 7\% no tenía relación con Ciencias de la Educación.

Según los estudios de Castellano (2017): en Ecuador solo se ofrecían 32 programas de postgrado en el área de la educación en todas las universidades nacionales el país. Esta oferta resultaba insuficiente para formar a los más de 150.000 profesores que trabajan en enseñanza inicial, básica y bachillerato (pág. 139-152).

Las carencias en la formación docente que afloran de los estudios nacionales desarrollados por el Instituto Nacional de Evaluación Educativa (INEVAL) en los exámenes "Ser Maestro", contemplan también la problemática para trabajar con la diversidad en el aula y lograr una educación inclusiva de calidad. Una forma de enfrentar estas carencias es a través de los estudios de cuarto nivel que como se ha explicado cuenta con un respaldo legal en toda la normativa ecuatoriana, es por ello que en función de diseñar una maestría en Educación Inclusiva se desplegó todo un estudio empírico en búsqueda de criterios de pertinencia.

\section{Metodología (materiales y métodos)}

Las razones metodológicos para la conformación de la población y la muestra, así como para la selección de los métodos y técnicas empleadas respondieron al criterio de explorar el conocimiento que tenían los profesionales incorporados al estudio acerca de la educación inclusiva, la información sobre la realidad existente en los respectivos centros educativos, 
sobre la necesidad de superación profesional, las expectativas de mayores oportunidades de empleo y de desarrollo profesional a partir de la maestría.

En tal sentido, se identificaron las siguientes categorías de análisis: conocimiento acerca del tema de la educación inclusiva, percepción de la autopreparación para desempeñarse en instituciones educativas inclusivas, conocimiento acerca de la diversidad y las discapacidades que presentan los niños, adolescentes y jóvenes de las instituciones educativas donde se encontraban laborando, barreras para lograr prácticas educativas inclusivas, interés por matricular la maestría y la importancia que le conceden al estudio del tema mediante una maestría.

Los criterios declarados se tuvieron en cuenta para construir la encuesta y la guía de los grupos de discusión con los estímulos discursivos que pudieran propiciar su debate para procurar que los temas y la secuencia de los mismos fluyeran de manera espontánea y no impuesta.

\section{Resultados (análisis e interpretación de los resultados)}

Análisis de los resultados de la aplicación de la encuesta sobre la pertinencia del nuevo programa de Maestría en Educación Inclusiva.

Gráfico 1. Lugar donde laboraban y formación que poseen los encuestados.

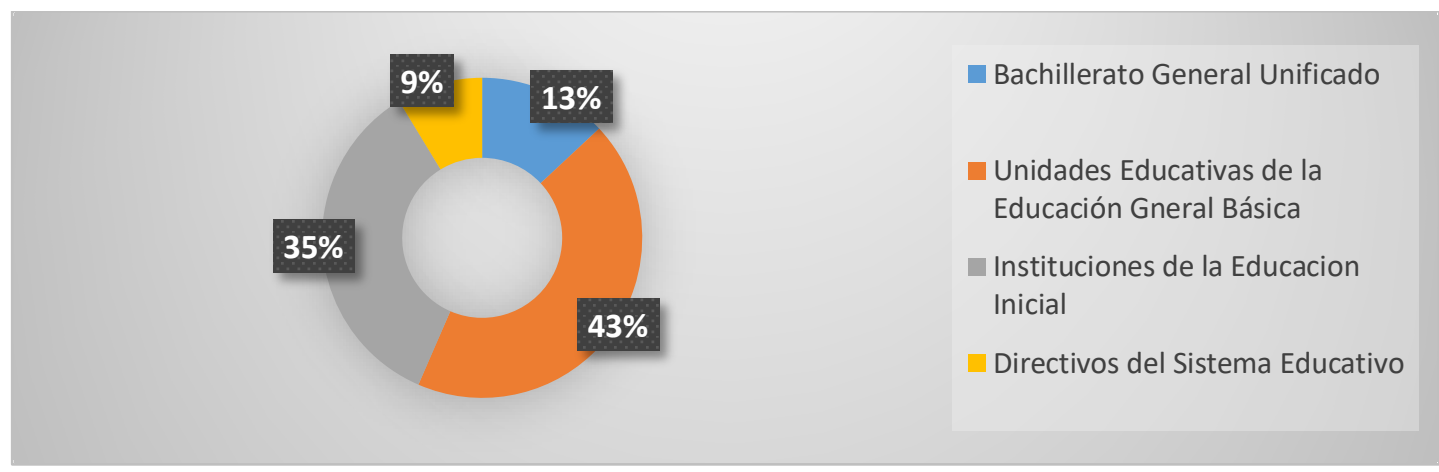

Fuente: Los Autores (2018).

Como se refleja en el gráfico 1, los 260 profesionales que 
voluntariamente entraron a la página de la Universidad y llenaron el instrumento que allí se había colgado trabajaban en: instituciones universitarias 30 , bachillerato 30 , unidades educativas de la educación general básica 100 , instituciones de la educación inicial 80 , directivos del sistema educativo 20, departamento de Consejería Estudiantil (DECE) y las Unidades Distritales de Apoyo a la Inclusión (UDAI) 20.

Gráfico 2. Formación que poseen los encuestados.

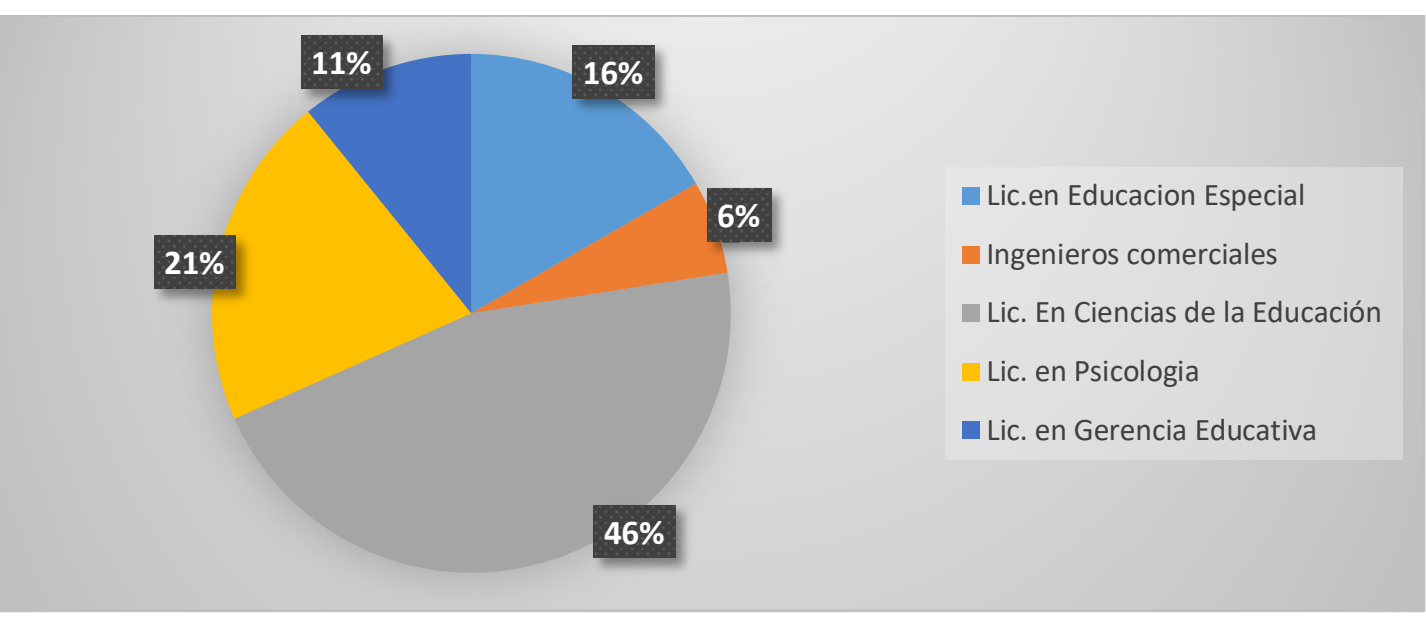

Fuente: Los Autores (2018).

Los participantes son graduados según refleja el gráfico 2, provienen de Bachilleres en Ciencias (20), Licenciados en Educación Especial (40), Ingenieros comerciales (14), Licenciados en Ciencias de la Educación (110), Licenciados en Psicología (50). Licenciados en gerencia educativa (26).

¿Si considera que es importante esta nueva Maestría en Educación Inclusiva?; ¿Si tenía interés en cursarla?. Ante estas interrogantes, los (260) participantes consideran que es pertinente y necesaria diseñar una maestría en educación inclusiva por lo que todos están interesados en matricularla. 


\subsection{En caso de ser su respuesta positiva, explicar brevemente la(s)} razón(es) que lo motivan a cursarla:

Las razones que los motivan a cursarla son:

- Con la finalidad de asegurar la permanencia de los estudiantes en el sistema educativo; por lo que considero de gran importancia el estudio a fondo en el tema, para desarrollar de manera eficiente el proceso de enseñanza aprendizaje.

- Es importante tomar en cuenta a aquellas personas que son iguales a todos, pero que tienen algo que las hace diferentes, y especiales.

- En el campo de la educación los docentes debemos ser innovadores, creadores de ambientes para que los estudiantes puedan resolver problemas de la vida cotidiana.

- Adquirir conocimientos y aplicarlos en las actividades diarias en bien de los estudiantes y la sociedad en su conjunto.

- Necesito mejorar mi formación profesional, ya que como maestra parvularia necesito que mi maestría tenga la línea de la inclusión y la adaptación curricular.

- Desarrollar, impartir, conocimientos, experiencias, destrezas y habilidades adquiridas durante mi trayecto profesional y laboral a la juventud actual.

- Formación personal, nuevas estrategias para la educación inclusiva.

- Me interesa mucho la maestría en educación inclusiva, ya que al ser docente las necesidades de cada día en las escuelas son prioritarias para poder ayudar a todos los estudiantes con N.E.E.

- Me interesa mucho la maestría en educación inclusiva, ya que al ser docente todos los días se tienen retos en las aulas educativas, por lo que creo que es necesaria la implementación de nuevas metodologías y medios de comunicación para ayudar a los estudiantes. 
- Integrar, ayudar y saber llevar a todos los niños, ya que todos tenemos los mismos derechos

- Porque he sido docente de personas sordas y a pesar de no tener una licenciatura en educación especial, estoy muy interesada en especializarme cada vez más en esa línea.

- Necesitamos formación de calidad para mejorar las capacidades de los niños con necesidades educativas, es muy importante capacitarnos en las diferentes áreas de la pedagogía para fortalecer los conocimientos previos y saber actuar de mejor ante los diferentes desafíos de nuestra carrera.

- Por el momento se ha avanzado en parte para que se de este cambio, pero aún no existe un personal capacitado, es decir docentes que estén enfocados en una educación inclusiva, actualmente se está formando docentes en educación especial, del cual tengo la oportunidad de formar parte. y me gustaría que el Ecuador ya cuente con un personal preparado y que mejor si somos nosotros los que vayamos a cursar una maestría en educación inclusiva y ser los pioneros para futuras generaciones.

- Para mi desarrollo profesional y así alcanzar un grado superior académicamente y ser un aporte en la sociedad.

- Para poder continuar con mis estudios y seguirme preparándome, pues al acabar mi carrera de educación especial deseo hacer una maestría y me gustaría mucho que se abra una maestría que se enfoque educación inclusiva.

- En la sociedad actual se han presentado casos de estudiantes con necesidades educativas especiales asociadas o no a la discapacidad, la cual los docentes o profesionales externos a los establecimientos educativos no tienen conocimientos para abordar estos casos en el 
aula, por tanto, es muy importante ser conocedora y ampliar mis conocimientos para poder abordar con seguridad y ejecutar estrategias pedagógicas que puedan ayudar y apoyar a los estudiantes de nuestra patria.

- Mi interés es porque nos permite ofrecer una educación de calidad tomando en cuenta las exigencias actuales de la sociedad, adaptándolas de acuerdo a las necesidades de cada estudiante con las diferentes estrategias metodológicas que permitan un aprendizaje significativo. el docente debe actualizarse constantemente donde permitirá interactuar con los estudiantes y realizar una clase motivadora.

Gráfico 3. Contenidos curriculares que necesitaban recibir en la maestría para fortalecer su formación en educación inclusiva.

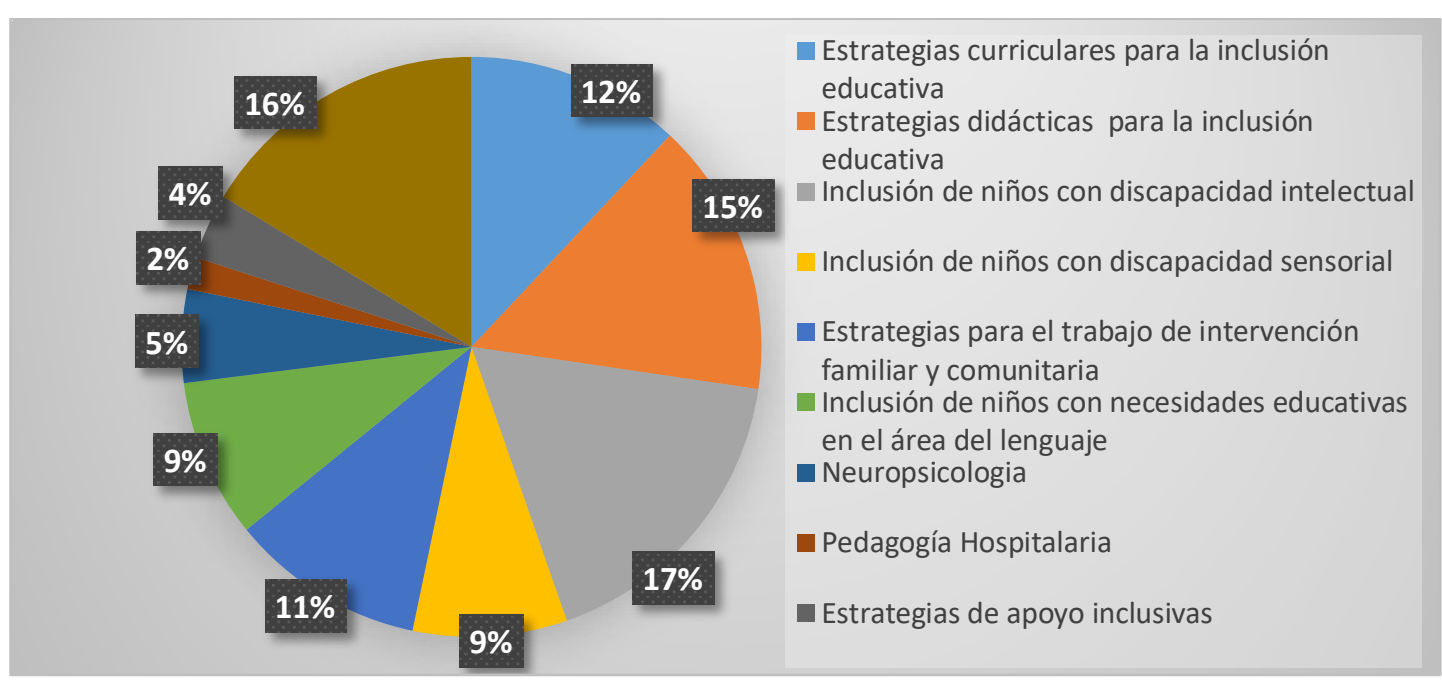

Fuente: Los Autores (2018).

Dentro de los contenidos curriculares que necesita recibir en la maestría, mencionados en el gráfico 3, para fortalecer su formación en educación inclusiva, los 260 encuestados declaran lo siguiente: estrategias 
curriculares para la inclusión educativa (180); estrategias didácticas para la inclusión educativa (230); inclusión de niños con discapacidad intelectual (260); inclusión de niños con discapacidad sensorial (129); estrategias para el trabajo de intervención familiar y comunitaria (63); inclusión de niños con necesidades educativas en el área del lenguaje (133); neuropsicología (78); pedagogía hospitalaria (27); estrategias de apoyo inclusivas (55); metodología de la Investigación educativa (245).

Gráfico 4. Bajo que modalidad de estudio le gustaría cursar la maestría.

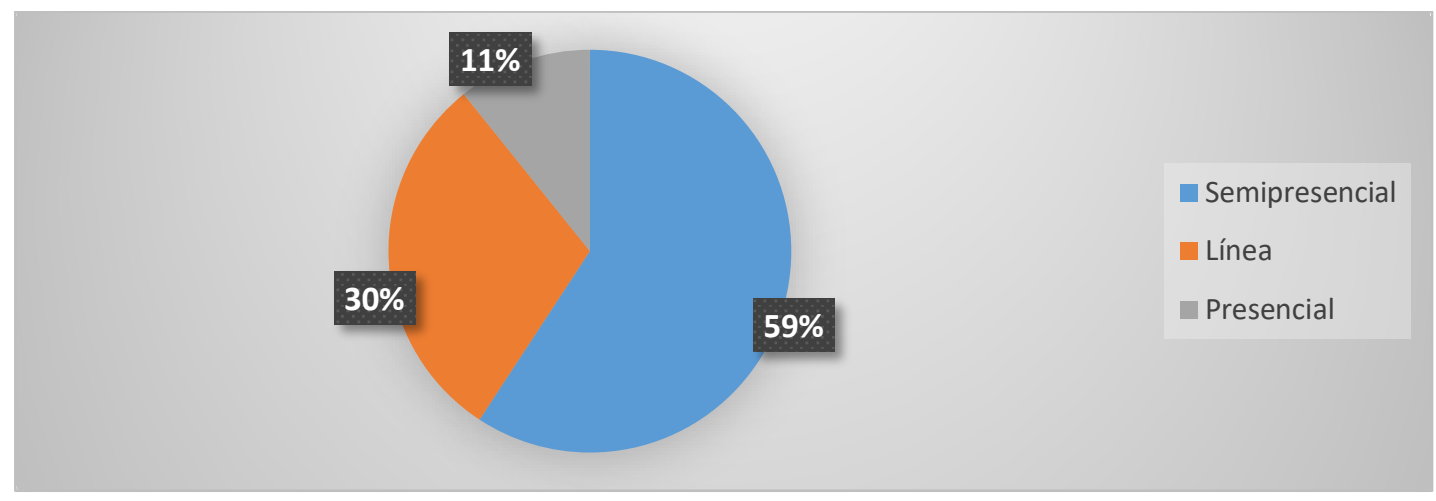

Fuente: Los Autores (2018).

Sobre la modalidad de estudio en la que les gustaría cursar la maestría, 154 encuestados dicen que prefieren cursarla semipresencial, 78 en línea y 28 presencial, como se indica en el gráfico 4.

\section{Análisis de los resultados que emergen de los grupos de discusión}

Se desarrollaron cuatro sesiones de los grupos de discusión con un total de 40 participantes, donde el debate giro alrededor de las siguientes interrogantes:

\subsection{Formación para la educación inclusiva en Ecuador}

Antes: Carencia de políticas y normativas que orientarán la educación 
especial tomando la iniciativa entidades privadas, ONGs, padres de familia, quienes se interesaron en la educación de los niños y niñas con dificultades, promoviendo la creación de centros de educación especial. Se asumen los acuerdos generados en el ámbito internacional respecto a la educación como derecho y se plantean lineamientos para la integración, se crean las aulas de apoyo.

Ahora: Se cuenta con la política pública y socializada que define y orienta la educación inclusiva en el Ecuador en las instituciones educativas públicas y privadas. Se plantean orientaciones para los currículos en las instituciones educativas y las adaptaciones curriculares para atender a los niños con NEE.

Se habla de flexibilidad curricular para atender la diversidad, sin embargo, se orienta desde la discapacidad. Se incluyen los conceptos de diversidad y vulnerabilidad.

\subsection{Formación en educación inicial, básica y media}

Antes: Inicialmente la educación se caracterizó por ser excluyente y segregadora y luego se avanzó hacia la integración. Los niños con NEE debían adaptarse a la escuela y el énfasis de los procesos era la debilidad con una educación homogeneizadora.

Ahora: Se demanda a todas las escuelas la inclusión, respaldados por la constitución política, los derechos humanos y la educación como derecho, las instituciones deben asumir a los niños con NEE, sin embargo, no se ha logrado un proceso formativo inclusivo. Se sigue apreciando una pedagogía terapéutica y previo diagnóstico se ubica a los niños por sus dificultades.

\subsection{Formación de formadores}

Antes: Capacitaciones y actualizaciones derivadas de la aparición de normativas y lineamientos del Ministerio de Educación, para ser aplicados en 
las IE, por tanto, coyunturales y descontextualizadas sin procesos de seguimiento, apoyo ni evaluación para verificar la comprensión y aplicación de las mismas. Las instituciones privadas asumen procesos de formación de docentes en temáticas específicas y se crean centros e institutos privados de formación, dentro de los que se encuentran las instituciones de educación superior privadas.

Ahora: Se cuenta con instituciones de educación superior públicas, que forman en la docencia en diferentes niveles, dentro de las que se encuentra la UNAE, para la formación de formadores que respondan a las necesidades educativas del país con enfoque inclusivo e intercultural, en los niveles de educación inicial y básica.

\subsection{Principales factores de exclusión a los que se enfrenta el sistema educativo ecuatoriano}

Los principales factores de exclusión se relacionan con el docente como eje central de los procesos formativos, la cultura, la accesibilidad, organización y socialización de información.

Factores relacionados con el docente: desconocimiento del marco conceptual y metodológico sobre los distintos tipos de diversidad (cultural, étnica, social...) y la inclusión; falta de comprensión a profundidad de las características diversas en los niños y niñas que le permitan al docente realizar una detección temprana de alguna dificultad; falta de formación y capacitación docente para abordar pedagógicamente procesos formativos inclusivos; el pensamiento y la praxis del docente que no se corresponde con los requerimientos para una educación inclusiva (flexibilidad, apertura e innovación); el compromiso del docente para asumir procesos inclusivos (aceptación, actitud positiva y motivación) y los perfiles de algunos docentes son profesionales en otras disciplinas y no evidencian conocimientos pedagógicos. 
Factores relacionados con la cultura: contradicción entre la cultura de la comunidad educativa institucional (familias, estudiantes, docentes y directivos) y las normativas estatales; la poca o nula participación de las familias en los procesos formativos de la Escuela.

Factores relacionados con la accesibilidad: las barreras arquitectónicas de la mayoría de las escuelas que dificultan el acceso de los estudiantes con algún tipo de dificultad; las barreras arquitectónicas de la misma ciudad; las barreras sociales y laborales que no permiten una continuidad de la formación del estudiante con NEE ni su desempeño laboral.

Factores relacionados con la organización y sistematización de la información: las plataformas del Ministerio de Educación que se habilitan para registrar la información sobre el desempeño de los estudiantes no cuentan con espacios para registrar información cualitativa sobre los niños que evidencian NEE; la falta de sistematización y actualización de la información de estudiantes de los distintos niveles educativos, que permita el acceso al mismo para articular y conectar los procesos que se adelantan y la necesidad de realizar un trabajo interinstitucional.

Aspectos de la formación y/o capacitación que pueden potenciar una transformación del magisterio ecuatoriano hacia una praxis más inclusiva, se declaran: el trabajo en equipo, la investigación educativa, el desarrollo de habilidades sociales y de comunicación, el desarrollo de proyectos de innovación, los proceso de detección temprana de necesidades educativas, las estrategias para aplicar la inclusión en el aula, el diseño de procesos de enseñanza-aprendizaje en el marco de la educación inclusiva, profundizar en los fundamentos psicológicos, pedagógicos de los modelos pedagógicos y el abordaje curricular.

Necesidades de formación profesional que experimentan u observan en la educación inclusiva, los participantes mencionan: capacitación y actualización en procesos investigativos, trabajo en equipos interdisciplinarios 
en el DECE, conocimiento sobre las Necesidades Educativas Especiales (NEE), comprender la política pública, preparación para asumir la educación inclusiva.

Competencias que serían necesarias para poder cubrir las carencias experimentadas: trabajo colaborativo, trabajo en equipos, capacidad investigativa y para trabajar con diversos actores, capacidad para la detección temprana de NEE, para realizar adaptaciones del currículo y del sistema de evaluación de los aprendizajes, generar ambientes inclusivos, trabajo de docentes en equipos multidisciplinarios e interdisciplinarios.

\section{Consideraciones Finales}

El estudio de pertinencia realizado para diseñar la maestría en Educación Inclusiva de la Universidad Nacional de Educación de Ecuador, se estructuró a partir de dos momentos fundamentales: el análisis de las políticas públicas y en particular las políticas educativas, y un el trabajo de campo, para conocer la opinión de los docentes, asesores y directivos para determinar las demandas que en el campo de la educación inclusiva realizaban.

El análisis de la situación educativa ecuatoriana permitió constatar que se producen importantes transformaciones, donde existe un consenso y orientación hacia la pedagogía de la inclusión y la atención a la diversidad de todas las personas en la escuela, pero se necesitan tomar medidas para que la misma sean más sólidas y sostenidas, precisando una mayor coherencia, consenso y aceptación social y ciudadana.

Es necesario reforzar la formación de cuarto nivel de los docentes a partir del estudio de pertinencia en los contextos en los que se puede desarrollas su actividad pedagógica (instituciones de la educación inicial, básica, instituciones especializadas, servicios de asesoramiento y apoyo educativo o socio-laboral, aulas hospitalarias, etc.).

Se pudo concluir, que existían condiciones favorables para la 
construcción de un ambiente de profesionalización docente a nivel de país comprometido con la educación inclusiva y la atención a la diversidad (conscientes de la diversidad humana, de las situaciones de riesgo y vulnerabilidad en la escuela y en la sociedad) que se podían lograr a través de propuestas formativas de cuarto nivel que conviertan la educación inclusiva en motor del cambio y trasformación social.

El análisis de las demandas del profesorado permitió configurar las competencias curriculares y el perfil de los egresados al que debía responder el diseño del programa de maestría y apuntó a lo pertinente de hacer una propuesta que respete los principios de la igualdad de derechos, consideran la diversidad como oportunidad para organizar respuestas a través de estrategias, recursos, metodologías y procedimientos colaborativos, cooperativos y participativos, reforzando la cultura de las prácticas inclusivas.

\section{Referencias}

Albornoz, O., (1996). Conferencia Regional sobre Políticas y Estrategias para la Transformación de la Educación Superior en América Latina y el Caribe: ideas, problemas y propuestas. Caracas, Venezuela: Centro Regional para la Educación Superior en América Latina y el Caribe (CRESALC); Organización de las Naciones Unidas para la Educación, la Ciencia y la Cultura (UNESCO). Recuperado de: https://unesdoc.unesco.org/ark:/48223/pf0000219410

Burgos, B., Rodríguez, A., \& López, K. (2011). Estudio de pertinencia de la oferta educativa de la Universidad de Sonora. Colección: Documentos de investigación educativa. ISBN de la colección: 978-6077782-55-1; ISBN de la obra: 978-607-7782-82-7. México: Universidad de Sonora. Recuperado de: http://docplayer.es/18420331-Colecciondocumentos-de-investigacion-educativa.html

Castellano, R. (2017). Pertinencia de la formación de postgrado en 
educación en el Ecuador. RUNAE. Revista Científica de Investigación Educativa de la UNAE, (1), 137-153, ISSN: 2550-6846; e-ISSN: 25506854. Recuperado de:

http://runae.info/index.php/RUNAE/article/view/13

Constitución de la Republica del Ecuador (2008). Título VII. Régimen del Buen Vivir. Capítulo Primero. Inclusión y Equidad. Registro Oficial 449 de 20-oct-2008. Ecuador: Asamblea Nacional Constituyente. Recuperado de:

https://www.oas.org/juridico/pdfs/mesicic4 ecu const.pdf

Crowe, E. (2010). Measuring What Matters. A Stronger Accountability Model for Teacher Education. Washington, D.C., United States: Center for American Progress. Recovered from:

https://cdn.americanprogress.org/wp-

content/uploads/issues/2010/07/pdf/teacher accountability.pdf

Darling-Hammond, L. (2006). Assessing Teacher Education: The Usefulness of Multiple Measures for Assessing Program Outcomes. Journal of Teacher Education, 57(2), 120-138, e-ISSN: 0022-4871. Recovered from: https://eric.ed.gov/?id=EJ735096

LOES (2018). Ley Orgánica de Educación Superior. Título VI. Pertinencia. Capítulo 1. Del Principio de Pertinencia. Registro Oficial Suplemento 298 de 12-oct.-2010. Quito, Ecuador: Asamblea Nacional Constituyente. Recuperado de:

https://www.epn.edu.ec/wp-content/uploads/2018/08/LOES.pdf

Malagón, L. (2003). La pertinencia en la educación superior. Elementos para su comprensión. Revista de la Educación Superior, 32(127), 113134, ISSN: 0185-2760; e-ISSN: 2395-9037. Recuperado de:

http://publicaciones.anuies.mx/revista/127/4/1/es/la-pertinencia-en-laeducacion-superior-elementos-para-su-comprension

Martínez, O., Rodríguez, M., \& García, W. (2018). Educación Continua de la 
UNAE: Un modelo que aporta a la Transformación Educativa del Ecuador. Revista Scientific, 3(8), 159-180, e-ISSN: 2542-2987. Recuperado de:

https://doi.org/10.29394/Scientific.issn.2542-2987.2018.3.8.8.159-180 OREALC/UNESCO (2013). Antecedentes y criterios para la elaboración de políticas docentes en América Latina y el Caribe. ISBN: 978-92-3001224-3. Santiago, Chile: Oficina Regional de Educación para América Latina y el Caribe; Organización de las Naciones Unidas para la Educación, la Ciencia y la Cultura. Recuperado de:

https://unesdoc.unesco.org/ark:/48223/pf0000223249

Tünnermann, C. (2006). Pertinencia y calidad de la educación superior. Lección Inaugural. Guatemala: Editorial: Universidad Rafael Landívar. Recuperado de:

http://iep.udea.edu.co:8180/entornoPGU/bitstream/123456789/258/1/P ertinencia\%20Calidad\%20Educacion $\% 20$ Superior $\% 20$ -

CarlosTunnermann.pdf

UNESCO (1995a,b). Documento de política para el cambio y el desarrollo en la Educación Superior. Código del documento: ED.94/WS/30. París, Francia: Organización de las Naciones Unidas para la Educación, la Ciencia y la Cultura. Recuperado de:

https://unesdoc.unesco.org/ark:/48223/pf0000098992 spa

UNESCO (1998). Conferencia Mundial sobre la Educación Superior en el siglo XXI: Visión y acción. Código del documento: ED.98/CONF.202/CLD.49. París, Francia: Organización de las Naciones Unidas para la Educación, la Ciencia y la Cultura. Recuperado de: https://unesdoc.unesco.org/ark:/48223/pf0000116345 spa 


\section{José Ignacio Herrera Rodríguez}

e-mail: joseighr2015@gmail.com

Nacido en Sancti Spíritus, Cuba, el 1 de septiembre del año

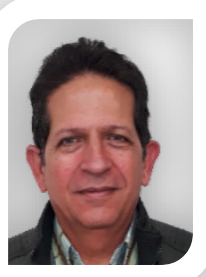
1961. Doctor en Ciencias Pedagógicas (PhD); Master en Ciencias de la Educación; Licenciado en Psicología; y Licenciado en Educación Especial; Catedrático de la Universidad de Sancti Spíritus (UNISS) en el periodo 1990 al 2014; Profesor Principal I de la Universidad Nacional de Educación (UNAE), en Ecuador desde el año 2015 al 2019, donde se me desempeñado como Director de la carrera de Educación Especial y actualmente Vicerrector de Investigación y Postgrado; Poseo amplia experiencia en el diseño de programas de grado, postgrado y en la formación de masters y doctores en ciencias. 


\section{Geycell Emma Guevara Fernández}

e-mail: geycellgf@gmail.com

Nacida en Sancti Spíritus, Cuba el 8 de agosto del año 1970.

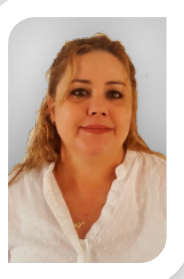

Doctora en Ciencias Pedagógicas; Máster en Ciencias de la

Educación; y Licenciada en Educación en la especialidad

Español-Literatura, por la Universidad de Sancti Spíritus

(UNISS), Cuba; Profesora no titular agregado 3 de la Universidad Nacional de Educación (UNAE) en Ecuador;

Tengo 25 años de experiencia docente, de ellos 15 dedicados a la Educación Superior en programas de pregrado y postgrado; He participado y dirigido varios proyectos de investigación en temas de orientación educativa, tutoría, didáctica, educación inclusiva y redacción académica. 


\section{Graciela de la Caridad Urías Arbolaez \\ e-mail: gracielauriasarbolaez@yahoo.es}

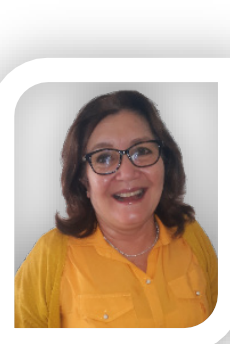

Nacida en Trinidad, Cuba, el 9 de noviembre del año 1960. Poseo amplia experiencia como docenteinvestigadora desde la enseñanza media hasta la educación superior, en universidades de Cuba y otros países de América Latina, como Brasil, Venezuela, y Ecuador; He dedicado más de 20 años a la formación de docentes; Vicedecano Docente; Vicerrectora Docente; Directora del Departamento de Psicología-Pedagogía; Directora de proyectos de investigación; Directora de tesis de PhD. y Maestrías; miembro del tribunal de grado científico para PhD. en Ciencias pedagógicas; Actualmente soy profesora con nombramiento y titularidad como Personal Académico Titular principal 1 de la Universidad Nacional de Educación (UNAE), en Ecuador y directora de la carrera de Educación Especial.

El contenido de este manuscrito se difunde bajo una Licencia de Creative Commons ReconocimientoNoComercial-Compartirlgual 4.0 Internacional 